\title{
Talking Through It
}

\section{Using Student-to-Student Interviews and Brainstorming Activities for Facilitating Critical Inquiry}

Krista Schmidt•Western Carolina University•kschmidt@wcu.edu

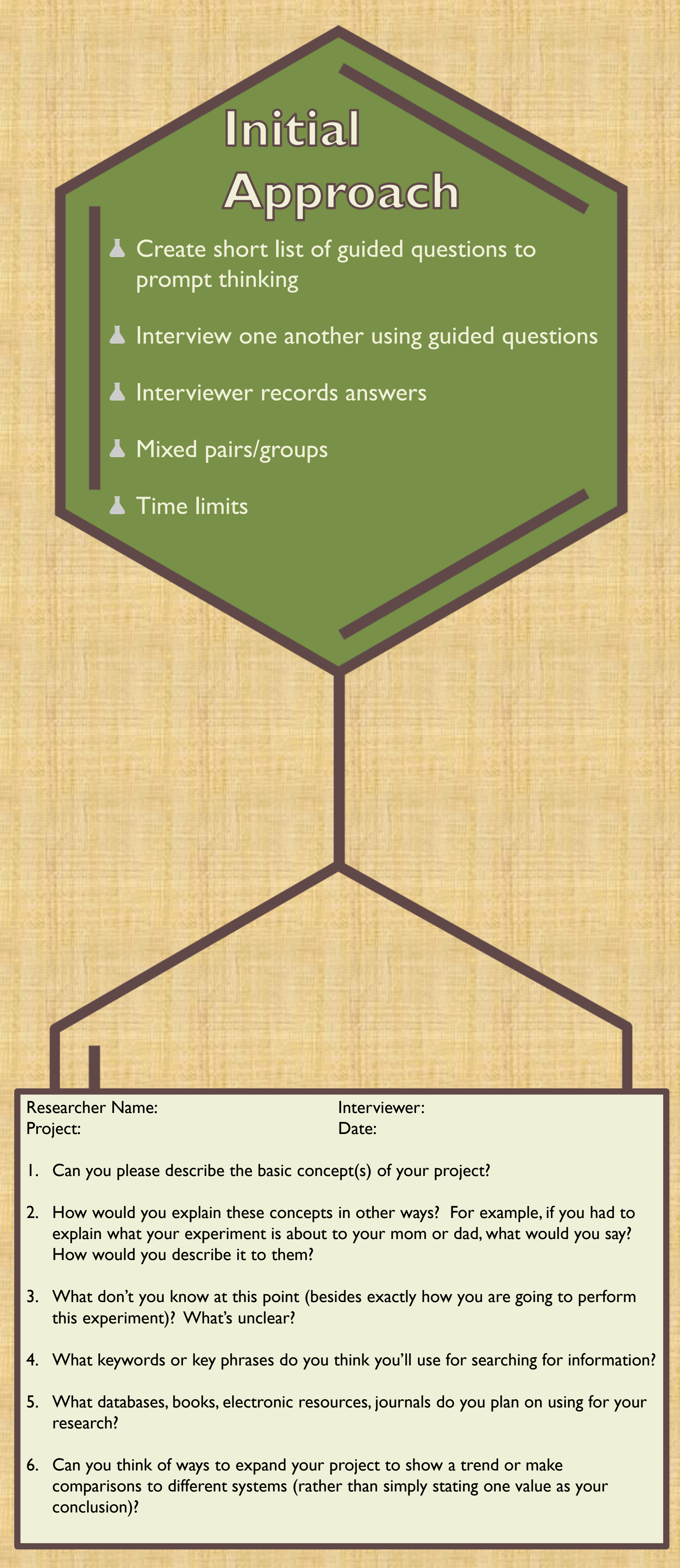

Outcomes and Observations

Questions as thinking prompts served as refreshers and Questions as thinking
search starting points.

Interviewees not distracted by writing "correct" or expected answers.

Interviewer occasionally provided additional topic information All project members, upon regroup, could contribute; members, professor, librarian then identified areas of topic (un)familiarity. Some questions too open-ended or vague; students struggled with "What do you mean?" Interviewers had trouble clarifying.

Few clarifications asked when the response was "I do not know." Amount of information collected varied.

Unfamiliarity with resources made source question too difficult.
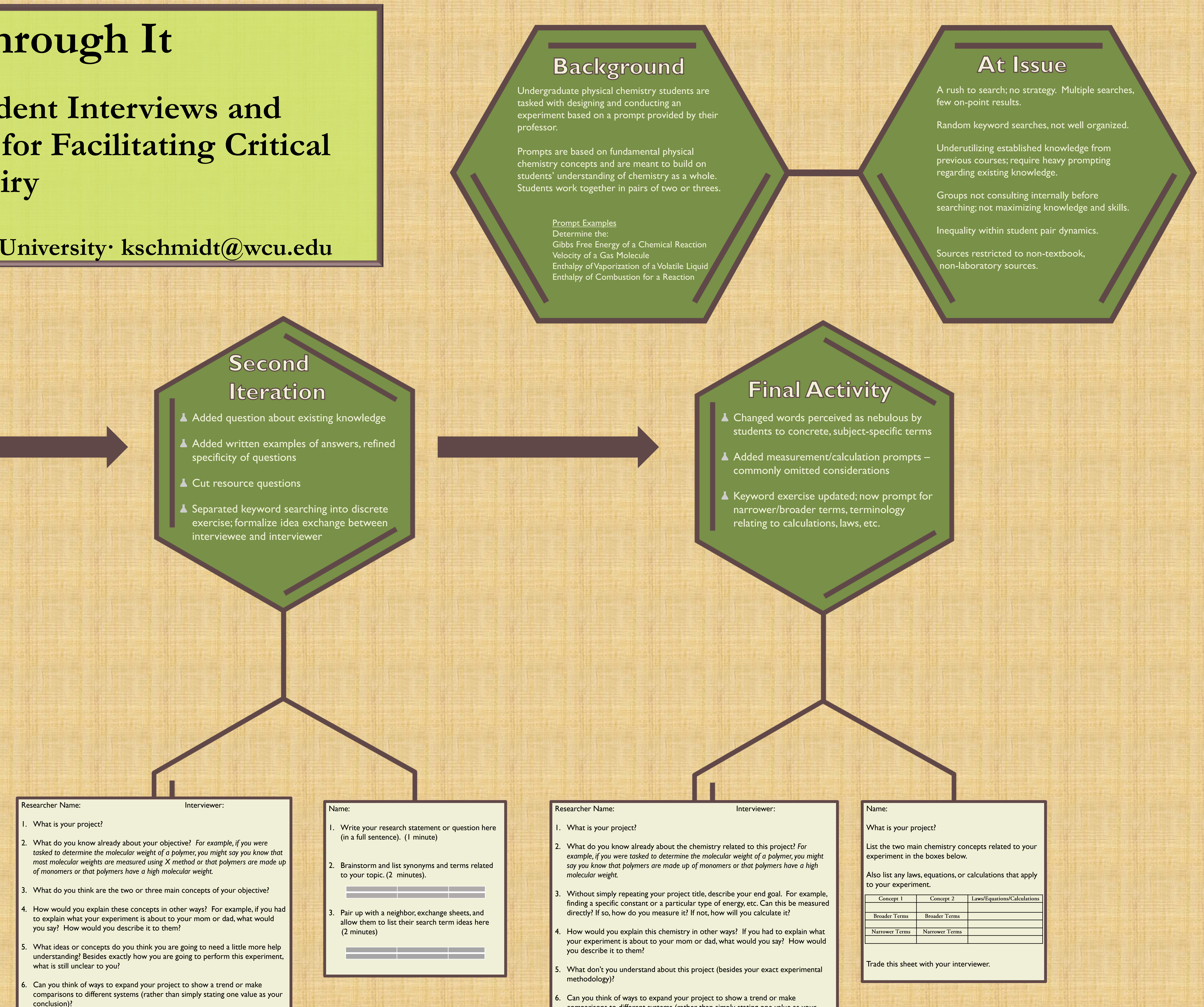

\section{Outcomes and Observations}

Questions about existing knowledge, with examples, equaled more productive interview.

Wording specificity resolved some vagaries and confusion about exact nature of question.

Addition of separate exercise and structured table encouraged critical thinking and served as better reference for search keywords.

Exchanging sheets addressed unequal feedback from interviewers.

Some questions still interpreted as too vague; students struggled to understand question rather than focus on ideas/concepts related to project.

Students often neglected fundamental considerations of project, particularly measurements, calculations, governing laws.
Final Acteivity

$\triangle$ Added measurement/calculation prompts -

$\Delta$ Keyword exercise updated; now prompt for
narrower/braader terms, terminology relating to calculations, laws, etc.

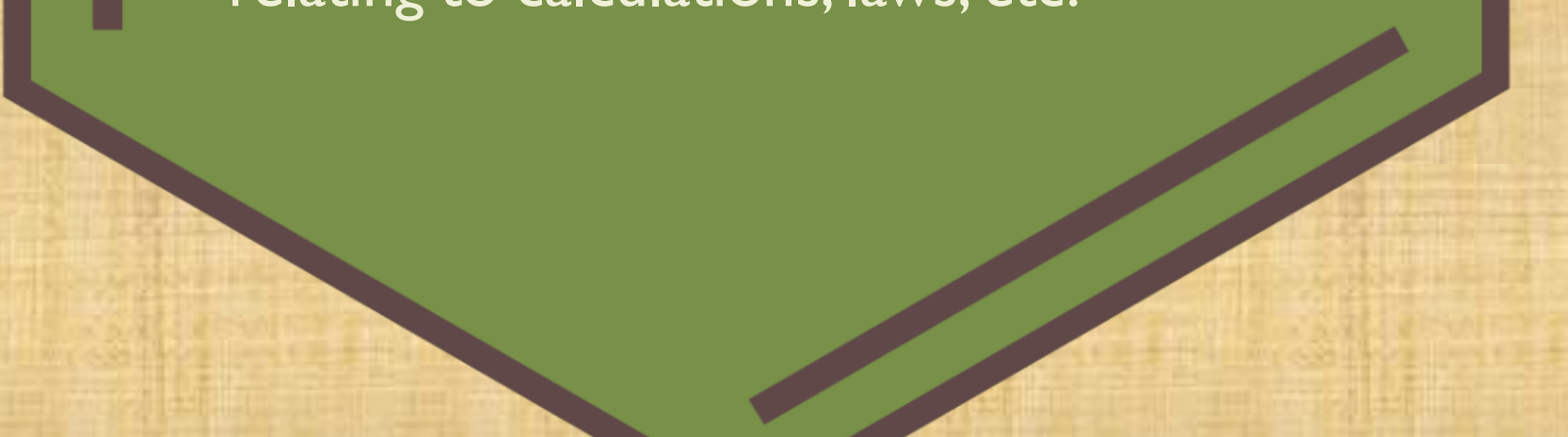

Researcher Name:

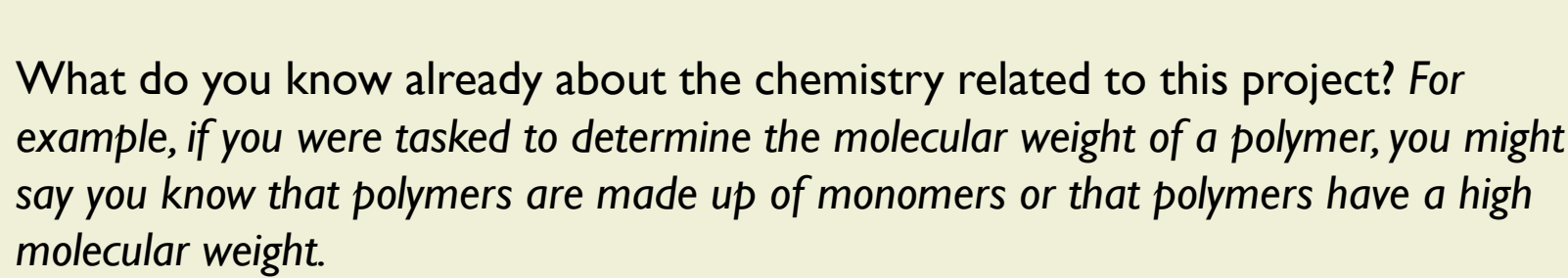

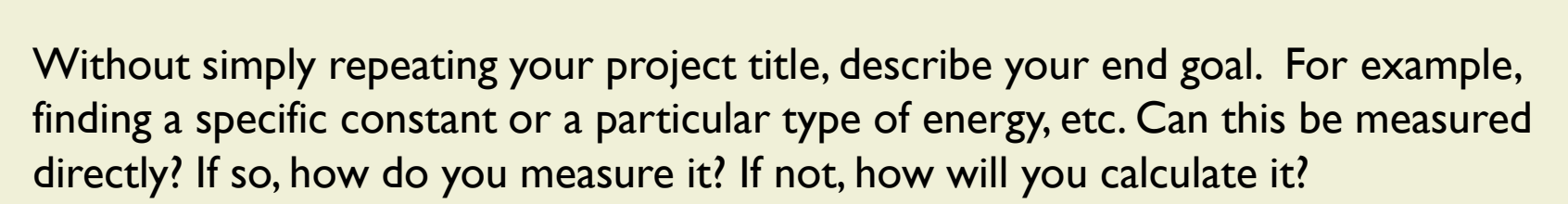

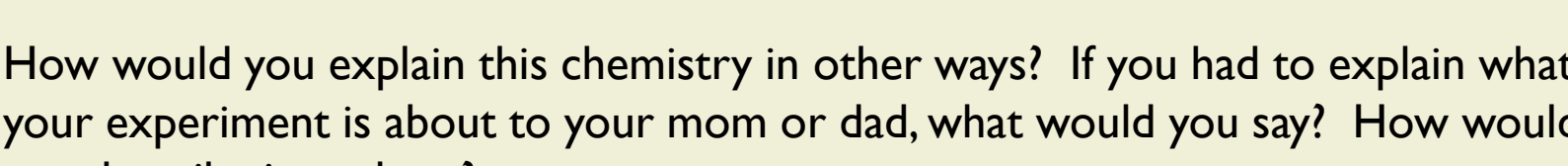

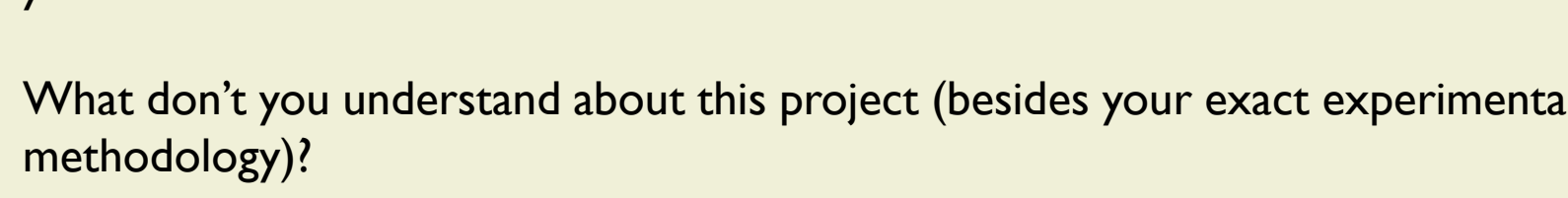

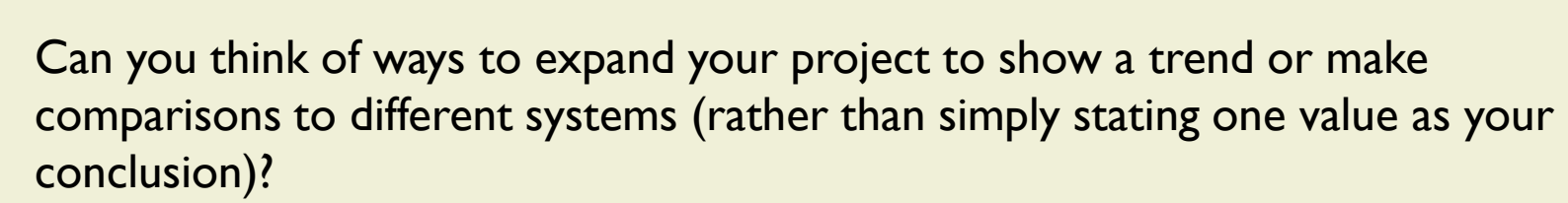

\section{Outcomes and Observations}

Increasing specificity of question terminology greatly reduced "What do you mean?" responses.

Forcing students to consider laws and measurements before initial design resulted in better design, more on-point searching strategies. Also pushed existing knowledge to forefront for consideration. of project while prompting recall of related chemistry concepts.

Specificity in interview and keyword exercise resulted in easier identification of actual inquiry problems. For example, do both group and individuals have thorough grasp of chemistry related to project? Are search keywords appropriate for each category?

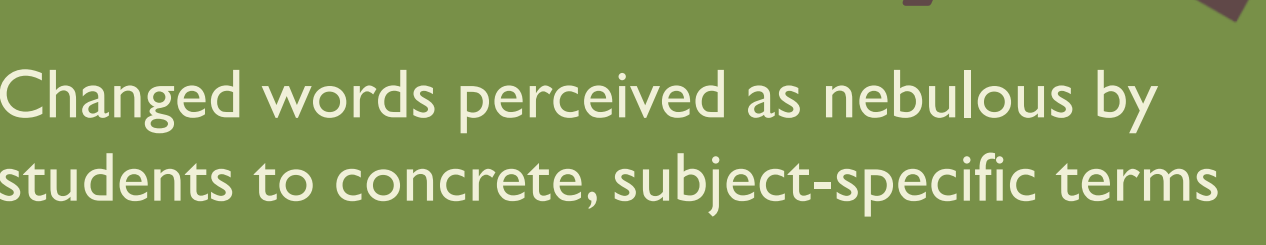

Keyword exercise redesign strategically pushed students to consider specificity 